\section{MS5-P35 Solution studies of human dipeptidyl peptidase III (hDPPIII) reveals dynamic equilibrium between different substates}

Prashant Kumar ${ }^{1}$, Henning Seidel ${ }^{2}$, Young-Hwa Song ${ }^{2}$, Christian Hübner ${ }^{2}$, Peter Macheroux ${ }^{3}$, Karl Gruber ${ }^{1}$

1. Institute of Molecular Biosciences, University of Graz, Graz 8010, Austria

2. Institute of Physics, University of Lübeck, Lübeck, Germany

3. Institute of Biochemistry, Graz University of Technology, Graz, Austria

email: prashant.kumar@uni-graz.at

Dipeptidyl peptidase III(DPPIII) is one of the most important enkephalin degrading enzymes associated with mammalian pain modulatory system.DPP III is also known to degrade several opioid peptides apart from enkephalin and endomorphin.Since the opioid peptides modulate large number of physiological processes, therefore DPP III is an important molecule to study.The crystal structure of the free form of the enzyme shows an open, extended conformation, whereas the crystal structure of the complex reveals a large conformational change upon peptide binding (Bezerra et al., PNAS, 2012).To investigate the behaviour of human DPPIII (hDPPIII) in solution we performed SAXS measurements which shows that this substrate triggered conformational change also occurs in solution. For the unbound form, however, the SAXS data indicate that the protein exists in either a mixture of fully open, fully closed or of other, different intermediate conformations. This conformational plasticity revealed by crystal structure and SAXS led us to perform single molecule flourescence resonance energy transfer(smFRET) with hDPPIII. SmFRET results show a broad range of energy transfer distribution between the two labels for the unbound form of the enzyme, with one maxima at low energy transfer corresponding to fully open conformation and another maxima at an intermediate closed conformation. For the bound form of the enzyme, two main maxima are observed, one at high energy transfer efficiency corresponding to the fully closed conformation and the other at a similar energy distribution as the intermediate closed conformation. These observations suggest the existence of equilibrium between different substates within the conformational space of the enzyme. Recently we were able to capture the crystal structure of the closed form of the enzyme in the absence of any ligand, however the crystal structure of the intermediate state still eludes us. The role of the intermediate state is unclear at the moment but this might just explain the promiscuity displayed by the enzyme. Our MD simulation data of $1.5 \mu \mathrm{sec}$ also indicates the existence of intermediate state which is in agreement with our experimental data. The insights gained by our structure determination and solution studies on hDPPIII provide valuable information about a common thread between structural and functional properties of enzymes and also the relation between the conformational dynamics and chemistry in enzyme catalysis.

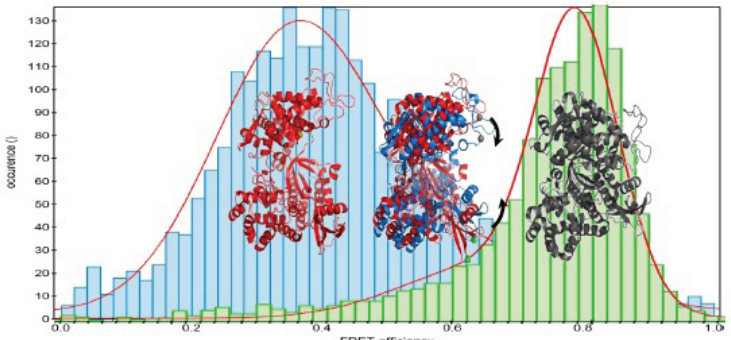

Figure1: Energy transfer histogram of human DPP III from smFRET. Energy transfer efficiency histogram of unbound in (blue) and bound (green). Crystal structure of fully (ben) ,fully closed(black) and intermediate structure obtained from MD simul (1) fully open structure(red): all three stu .

Figure 1. Energy transfer efficiency histogram of hDPP III from smFRET and structures corresponding to their energy transfer efficiency

Keywords: Conformational plasticity, Opioid peptides, Peptidase, Single molecule, SAXS 